\title{
Author Correction: Genome-wide meta-analysis of phytosterols reveals five novel loci and a detrimental effect on coronary atherosclerosis
}

\author{
Markus Scholz (D), Katrin Horn (1D, Janne Pott (D), Arnd Gross (D), Marcus E. Kleber (D), Graciela E. Delgado,
} Pashupati Prasad Mishra (10, Holger Kirsten (1), Christian Gieger (1), Martina Müller-Nurasyid, Anke Tönjes, Peter Kovacs (1D), Terho Lehtimäki (1D, Olli Raitakari, Mika Kähönen, Helena Gylling, Ronny Baber, Berend Isermann, Michael Stumvoll (1), Markus Loeffler, Winfried März, Thomas Meitinger, Annette Peters (D), Joachim Thiery, Daniel Teupser \& Uta Ceglarek

Correction to: Nature Communications https://doi.org/10.1038/s41467-021-27706-6, published online 10 January 2022.

In this article the affiliation details for Helena Gylling were incorrectly given as Department of Internal Medicine, University of Helsinki and Helsinki University Hospital, Helsinki, Finland but this should have been Heart and Lung Center, Cardiology, University of Helsinki and Helsinki University Hospital, Helsinki, Finland. The original article has been corrected.

Published online: 25 February 2022

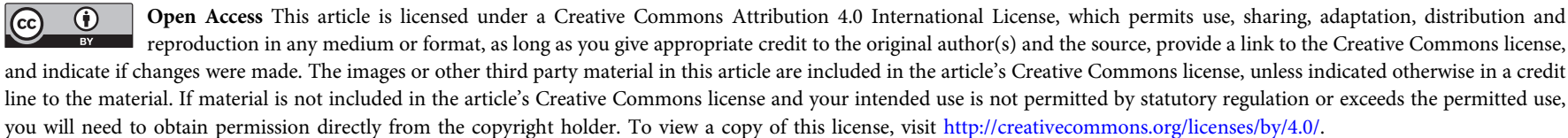

(c) The Author(s) 2022 